\title{
Spinal intramedullary cavernomas. Personal experience reffering to six cases
}

\author{
G. Iacob, Angela Olarescu \\ Neurosurgery Clinic, University Emmergency Hospital, Bucharest
}

Despite cavernous malformations of the CNS are pathologically similar, intramedullary cavernous malformations are very rare lesions, increasingly recognized after introduction of magnetic resonance image, generating gradual neurological decline, with severe deficits or acute loss of spinal function. We report our experience on six patients with intramedullary cavernomas defining the spectrum of presenting symptoms and signs analyzing the role of surgery as a treatment for these lesions. We present our experience with 2 cervical and 4 thoracal spinal intramedullary cavernoma from 2010 to 2014 searching history, onset of clinical manifestation, neurological status, radiological findings, operation, and clinical outcome. Among 6 patients male were 2 cases; female 4 cases; mean age was 42 years (range 25-72 years); mean duration of symptoms were 1,5 years (range 5 days and 2 years) with slowly progressive neurological decline. In two cases there was acute onset of neurological compromise. In all cases diagnosis was made on MRI and lesions were possible to be radically excised and gently extracted from the hemosiderin-stained bed inside of the spinal cord via a laminectomy and midline myelotomy with microsurgical techniques. The surgical outcome on a mean duration of follow up of 12 months were: for 4 cases - the patients neurological conditions remarkably improved 1 month later, for 2 cases no improvement were remarked. No recurrent hemorrhages were recorded. A follow-up MRI examination was made in all cases to confirm complete removal of the cavernous angioma. Spinal intramedullary cavernoma should be early recognized by MRI, can be positioned in a precarious position and generate significant neurologic deficits than cranial cavernomas. For symptomatic intramedullary cavernous malformations extended to the dorsal surface of the spinal cord, total resection with microsurgical techniques can offer good or excellent outcome, restoring neurological status and to stop chronic deterioration and acute rebleeding. To asymptomatic patients with deeper lesions which entail a higher operative risk, but also a surgically manageable cause of myelopathy a closed observation is mandatory.

Key words: intramedullary cavernous malformation, spinal MRI - magnetic susceptibility sequences, microsurgical resection. 


\section{Introduction}

Cavernous malformations are well-defined lesions composed of abnormally dilated blood vessels, vascular channels with structurally incomplete vessel walls, lined by a thin endothelium without intervening normal nervous tissue, surrounded by gliotic medullar tissue, often stained with hemosiderin, expresion of previous hemorrhage. Intramedullary spinal cord cavernous malformations are unusual: solitary or multiple lesions discovered in both the brain and spinal cord using MRI and justified by genetic basis $(1,2)$, causes neurological symptoms due to the higher density of eloquent structures in the spinal cord and have an unknown natural course (3-7).

\section{Methods and results}

We present our personal experience concerning 2 cervical (C3 and $\mathrm{C} 5$ ) and 4 thoracal (T2-T3, T6, T7 and T9) spinal intramedullary cavernoma from 2000 to 2014 . Among 6 patients male were 2 cases; female 4 cases; mean age was 42 years (range 25-72 years) at presentation; mean duration of symptoms were 1,5 years (range 5 days and 2 years) with slowly progressive neurological decline (stade 2 according to Ogilvy classification) (8) and symptoms (mild paraparesis, hypoesthesia, gait, trophic and sphincterian disturbances) evaluated on the Frankel scale. In two cases the onset of neurological compromise was acute: 3 hours respective 18 hours (stade 3 according to
Ogilvy classification). In all cases diagnosis was made on MRI and lesions were possible to be radically excised and gently extracted from the hemosiderin-stained bed inside of the spinal cord via a laminectomy and midline myelotomy with microsurgical techniques. The surgical outcome on a mean duration of follow up of 12 months were: for 4 cases

- the patients neurological conditions remarkably improved 1 month later, for 2 cases ( $\mathrm{C} 3$ and T2- T3) no improvement were remarked. No recurrent hemorrhages were recorded. A follow-up MRI examination was made in all cases to confirm complete removal of the cavernous angioma.

\section{Illustrative case:}

A 48-years-old, complained of thoracolumbar pains, bilateral leg weakness, especially on the left leg, legs numbness 1 year before admission, with sudden deteriorating legs weakness and urinary retention 2 weeks before admission. He presented with a BrownSequard syndrom: spastic paraparesis especially on left side, left Babinski and foot clonus signs, with impaired right thermo-algic sensation below the T8 dermatome.

The patient underwent cranial and spinal MR examinations and postoperative MR imaging in a 1,5-Tesla MR imager. Sagittal T1 and T2 - weighted images and axial T2 weighted MR images of the thoraco-lumbar spine reveal an intramedullary T6, 11/9 mm, well-marginated lesion, with heterogenous signal abnormality in both $\mathrm{T} 1$ and $\mathrm{T} 2$ weighted images, representing blood products 
DOI: 10.2478/romneu-2014-0056

of various ages. The lesion was surrounded by low signal intensity, representing iron storage products and medullary perilesional oedema, occupying most of the thoracic spinal cord, suggesting the diagnosis of an intramedullary thoracal cavernoma. Figure 1, Figure 2

After induction of general anesthesia, the patient was positioned prone. The patient underwent a T6-T7 laminectomy. The dura was sharply incised on the midline, reflected to expose the lesion and retained laterally using No 4-0 silk sutures between its edges and the paraspinous muscles. Using the operating microscope, the arachnoid was opened; the widened, brown colored spinal cord becomes apparent at the lesion level. A midline mielotomy to preserve the fine vascular network is performed, lacate blood was aspirated and an entirely intramedullary yellow- dark red mass, clearly demarcated from the adjacent spinal cord tissue, was excised and gently extracted from the the hemosiderin-stained bed inside of the spinal cord. After the lesion has been completely resected, the walls of the cavity remaining in the spinal cord collapse. The dura was closed in a watertight manner. The muscular, fascial, subcutaneous and cutaneous layers were closed in the standard fashion. The resected material showed thin-walled vascular spaces, variable in caliber and fibrous tissue content, occlusive thrombus attached to the vascular wall, revealing a cavernoma. (Figures 3-6.) The clinical course was uneventful: the patient was encouraged to begin ambulation the following morning and his neurological condition remarkably improved: 1 week later he recovered motor strength and sensation in his left foot and was able to walk without cane and climb stairs. A 3 day postoperative MRI showed a postoperative status, a little intramedullary zone with magnetic susceptibility effect, suggesting post operatory intramedullary hemorrhagic remnants at T6 level, surrounded by a minimal intramedullary oedema. At 1 month follow up, the patient's spasticity has diminished and he was able to walk without difficulty.

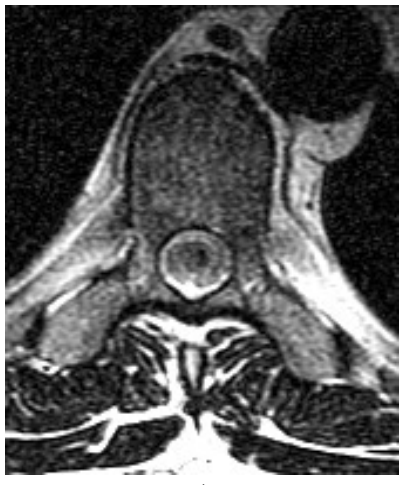

A

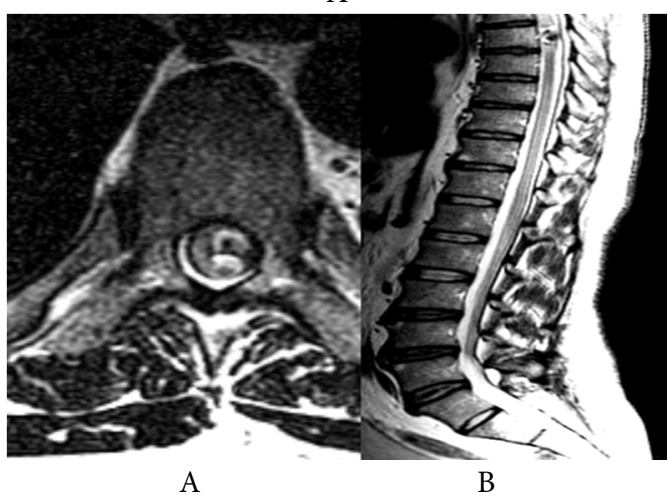

Figure $1 \mathrm{~A}$ - axial T2w images show low signal intensity on the lesion - susceptibility effect and hiperintense signal intensity in the perilesional edema; B - axial T1w images reveal mixt hiperintense and hipointense T1w signal, representing an intramedular lesion with methemoglobin contain, C - T6 spinal intramedular mixt signal lesion and perilesional edema of the spinal cord 


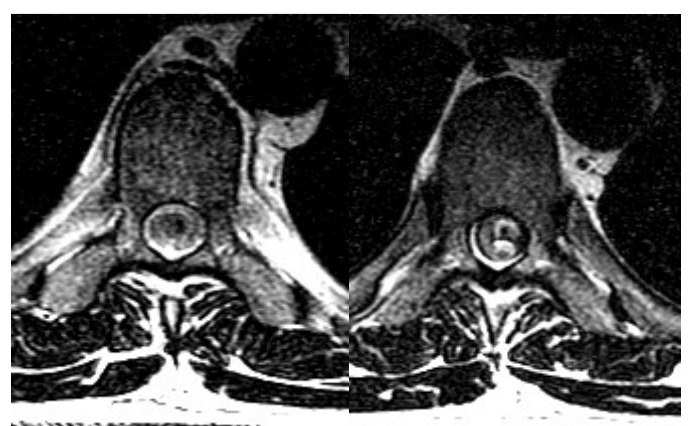

Figure $2 \mathrm{~A}$ - axial T2w images show low signal intensity on the lesion - susceptibility effect and hiperintense signal intensity in the perilesional edema B - axial T1w images reveal mixt hiperintense and hipointense T1w signal, representing an intramedular lesion with methemoglobin contain

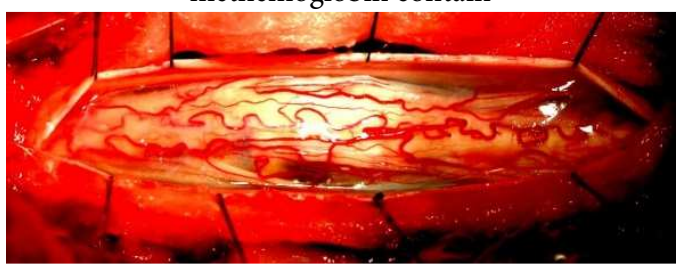

A

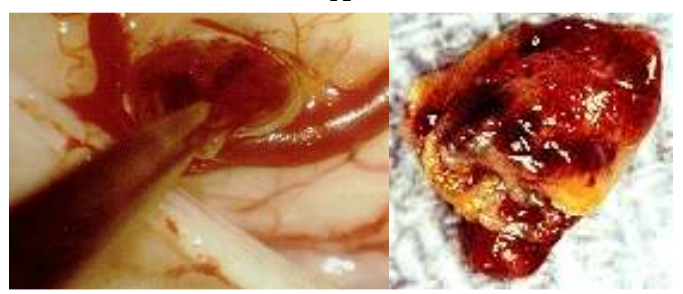

B

C

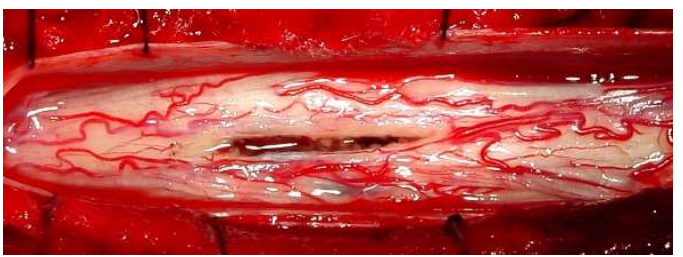

$\mathrm{D}$

Figure $3 \mathrm{~A}$ - the surface of the left medulla, at T6-T7, is widened, brown colored, from previous hemorrhages, making intramedullary cavernoma apparent, B - after a median myelotomy, lacate blood was aspirated and the cavernous malformation is sharply dissected from its surrounding bed, mobilized and removed, $\mathrm{c}$ the pathologic specimen is shown, $\mathrm{d}$ after the lesion has been removed completely, the walls of the cavity remaining in the

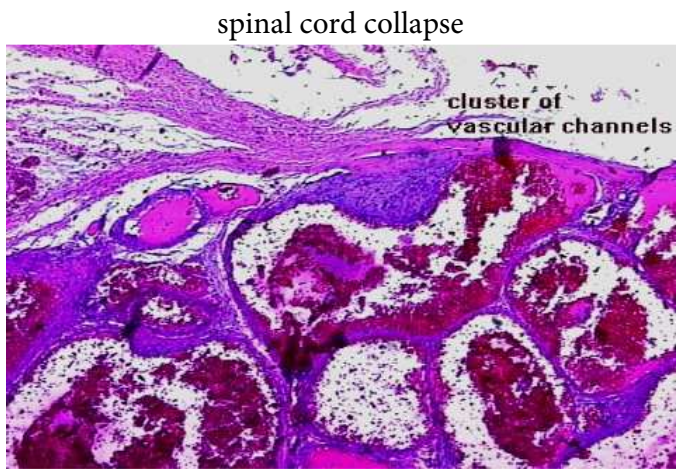

A

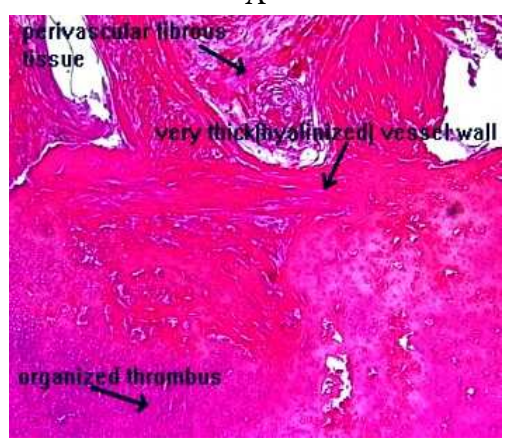

B

Fiure $4-\mathrm{H} \& \mathrm{E}$ staining a vascular spaces variable in caliber and fibrous tissue content; the extraluminal interstices are chinked with fibrous connective tissue, note no intermingled nervous tissue. Original magnification $\times 100 \mathrm{~b}$ detail of an occlusive thrombus attached to the vascular wall. Original magnification $\times 100$

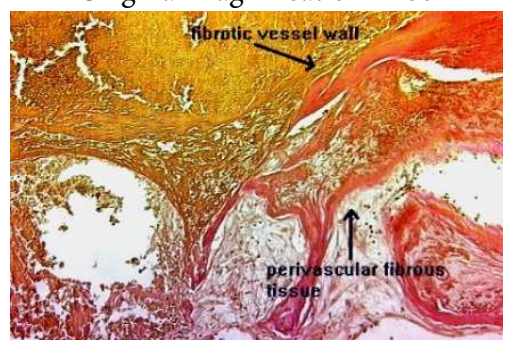

Figure 5 - Van Gieson staining.fibrosclerotic perivascular tissue, marked fibrosis in vascular wall, Original magnification x100 


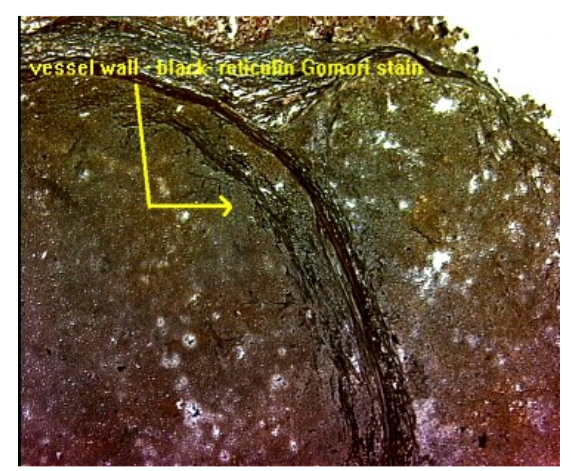

A

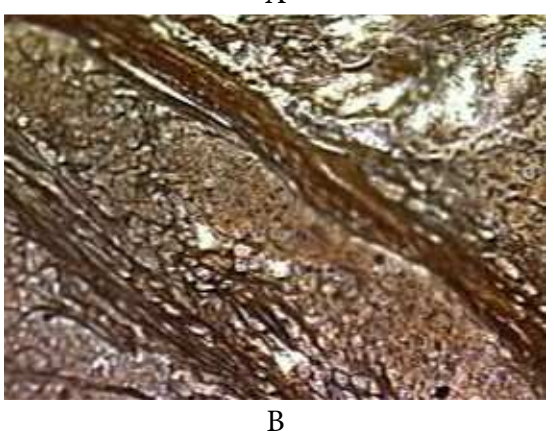

Figure 6 - Gomori staining: a fibrotic vascular wall; occlusive thrombus, Original magnification x100, b detail of fibrotic wall, Original magnification $\times 200$

\section{Discussion}

Cavernous angiomas also known as cavernous malformations, cavernous hemangiomas (9) or cavernomas occur throughout the central nervous system, representing $10 \%$ of all SNC vascular abnormalities are considered to be congenital hamartromas in contrast to true vascular neoplasms (3)(10). Although they are most commonly found in the brain, the intramedullar cavernoma accounts for approximately 5 to $12 \%$ of all adult intramedullary lesions in adults (11) and $1 \%$ of intramedullary lesions in children (12)(13). Usually solitary they may be associated with cavernous angiomas in other organs or in the central nervous system where they frequently originate in the vertebrae with occasional extension into the extradural space (14) (15).

The first spinal cavernoma was reported in a 27-year-old girl who died 212 days after an acute occurrence at 1901; Schultze reported the first total resection in 1912 and McCormick was first advocated with complete microscopic resection in 1988 (16)

Intramedullary cavernoma tend to become clinically symptomatic during the third and the fourth decade of life, like cerebral cavernous malformations (4), but they are more aggressive than cranial cavernomas, probably because the spinal cord is less tolerant of mass lesions. Reports of familial incidence and multiple localizations, involving both brain, spinal cord and other organs (skin, retina), suggest the possibility of genetic mechanisms in the development of such lesions (1) (2). They are more common in women - in two thirds of cases, in the cervical and thoracic regions of the spinal cord, as in our series (17). There is a male preponderance in pediatric patients (12) Intramedullary cavernomas are slow-growing lesions, the risk for hemorrhage range was estimated from 1.7 to $4.5 \%$, per year (3) (11) and may enlarge in three different ways which may coexist:

- small bleedings, (chronic hemorrhages) often clinically silent, which create a small cavity around the blood vessels, causing hemosiderin deposition in the surrounding neural tissue with reactive gliosis

- budding of new capillaries as reactive angiogenesis with new vessel formation, the nidus may grow over time, increasing the mass 
of the malformation, the so-called hemorrhagic angiogenic proliferation

- acute massive hemorrhage with rapid expansion and acute mass effect or repeated intralesional hemorrhage and thrombosis, with expansion of a hemorrhagic cyst cavity. Sometimes cavernomas are associated with cryptic venous malformations (18).

Histopathological features of intramedullary cavernomas are similar to those of cranial lesions (19) (20): -macroscopy: a dark red or purple spongy mass composed of vessels and blood on gross examination, surrounded by discolored glial tissue. The size is reported to vary between less than $1 \mathrm{~mm}$ up to more than $10 \mathrm{~mm}$ in diameter; -microscopy: vascular channels, blood-filled, with hyalinized thin walls, without elastica interna, closely apposed, abnormal venous channels, without intervening medullar tissue. Those cavernous malformations that lack calcification has a greather tendency to bleed.

Intramedullary cavernomas simptomatology (pain, weakness, paraesthesias, slowly progressive myelopathy, subarachnoid hemorrhage) (11) (21-23) are related on: tumor location with respect to the spinal topography, but also to the spinal cord; abnormal vessels which may lead to spinal cord compression; cord expansion or oedema, ischemia generated by arterial steal, repeated hemorrhage and venous hypertension. Our findings are similar to those of others (24) (25); several patterns of intramedullary cavernomas clinical presentation are known:

- asymptomatic, accidentally found at autopsy in patients with multiple cavernous angiomas;
- gradual slow neurological deterioration over months to years (8), with acute episodes lasting for hours to days, with possible neurological recovery between episodes generated by small hemorrhages or acute thrombosis with organization and recanalization

- mild neurological symptoms, with progressive myelopathy, the most frequent mode of presentation, of gradual decline during weeks to months or even acute onset, events related to changes in the microcirculation surrounding the lesions and to the neurotoxic effect of hemosiderin;

- acute complete paralysis, fortunately not common but seen in pediatric population (13), due to hemorrhage extension into the spinal parenchyma, similar to cauda equine cavernoma (26) where intratumoral microbleeding may generate acute onset.

Intramedullary cavernomas are occult angiographcally and often CT, but MRI is the current study of choice both for the diagnosis and for surgical planning, with particularly high sensitivity blood oxygenation level (27). Typical manifestation: "popcorn appearance" - a webbed core, composed of blood and blood products in various states of evolution of mixed spinal intensity on both $\mathrm{T} 1$ and $\mathrm{T} 2$ weighted images, with moderate, occasionally strong absorption of contrast medium after an infusion of gadolinium. As a sign of previous hemorrhage in the T1-weighted image, as well as in the T2-weighted image, a black ring of low signal intensity around the cavernoma, consistent with hemosiderin deposits from old hemorrhages indicates disturbances of susceptibility caused by the iron in 
DOI: 10.2478/romneu-2014-0056

hemosiderin. Blood products are identified depending the age of a hemorrhage: isointense to slightly hypointense on $\mathrm{T} 1$ weighted images and hypointense on T2 weighted images susceptibility effect. If edema is present, the signal outside the hemosiderin rim is increased on the T2-weighted images. Small cavernous malformations may appear only as petechial areas of decreased signal density, "black dots". Although large vessels are uncharacteristic of cavernous malformations, a coexistent large draining vein is occasionally identified (16). In the subacute hemorrhage stade, few days later, the lesion that contains methemoglobine are hyperintense on T1weighted images and hypointense on T2 weighted images (susceptibility effect). Several weeks later, in the hemosiderin chronic stade, old blood products are hypointense "blooming" on both T1 and T2 weighted images. Changes in volume and gradient echo (GE) MRI characteristics are frequent seen, that is the result of hemorrhage evolution. Small intramedullary cavernomas have usually no edema; differential diagnosis should rule out cryptic AVMs and hemorrhagic neoplasms. Even when small, with characteristic surrounding edema.

The natural history of untreated cavernomas (6) (28-30) is still unclear: after (23) intramedullary cavernomas tend to be clinically progressive, neither the indication for the optimal timing for surgery are known: it's preferable to operate intramedullary cavernoma in the subacute stage before rapide decline of symptoms and when gliosis surrounding lesion protects the normal spinal cord facilitating removal (5) (14). Early microsurgical en bloc resection should be considered for:

- asymptomatic intramedullary cavernous angiomas in children, for the potential devastating hemorrhage particularly significant (12)

- for all symptomatic patients, especially to those lesions that extend to the dorsal surface of the spinal cord, before enlargement or rebleeding; generating gradual clinical improvement or to halt the progressive neurological decline; prognosis are depending on the severity and duration of the preoperative status (31)(32).

A subtotal resection should be considered for deeper lesions (despite possible future hemorrhages with adverse consequences); when neurological function is at risk, since rebleeding of the residual lesion can occur.

To limit surgical trauma intraoperative ultrasonography is able to localize the hyperechogenic malformation, guiding the extent of myelotomy, also intraoperative somatosensory and motor- evoked potential monitoring must be used (11) (33). After dura opening, the presence of anomalous vascular reticule or swelling on pia mater could suggest an underlying hemorrhagic lesion. Using the shortest path approach midline cordotomy or a postero-lateral approach the wellcircumscribed dark-bluish lesion as the small low-flow feeding vessels should be identified and coagulated and lesion should be carefully dissected and removed in one piece. The outcomes of surgery for cavernous malformations are related to the preoperative neurological status and total removal of the lesion. Although patients could transiently 
experience neurological worsening after surgery, complete removal is generally possible with minimal morbidity. Also surgery cannot cure the chronic myelopathy from cavernoma, but can halt its progression. These facts emphasize the need for early diagnosis. Delayed complications were the result of incomplete resection (5) (18) (34) (35). The resultant hemorrhage: the reported risk is $9 \%$ (15) or $17,6 \%$ (18) required reexploration, which led to tethering of the spinal cord. Most patients who underwent resection, however, had improved neurologically at long-term follow-up.

\section{Conclusions}

Spinal intramedullary cavernoma can be positioned in a precarious position, may generate significant neurologic deficits than cranial cavernomas and should be early recognized by MRI For symptomatic intramedullary cavernous malformations extended to the dorsal surface of the spinal cord, total resection with microsurgical techniques can offer good or excellent outcome, restoring neurological status and stop chronic deterioration and acute rebleedng. For asymptomatic patients with deeper lesions entail a higher operative risk, also a surgically manageable cause of myelopathy a closed observation is mandatory. Similar residual lesion possible masked by surrounding gliosis impose a careful follow up.

\section{References}

1.Cohen-Gadol AA, et al. - Coexistence of intracranial and spinal cavernous malformations: a study of prevalence and natural history, J Neurosurg 2006, 104:376-381

2.Lee S.T. et al. - Identification of an Arg35X mutation in the PDCD10 gene in a patient with cerebral and multiple spinal cavernous malformations, J Neurol Sci 2008, 267:177-181

3.Kondziella D. et al. - Cavernous hemangioma od the spinal cord - conservative or operative management? Acta Neurol Scand 206, 114: 287-290.

4.Santoro A. et al. - Intramedullary spinal cord cavernous malformations: report of ten new cases, Neurol Rev. 2004, 27: 93-98

5.Aoyama T. et al. - Intramedullary Cavernous Angiomas of the Spinal Cord: Clinical Characteristics of 13 Lesions, Neurol. Med. Chir. (Tokyo) 2011, 51, 561-566

6.Kharkar S. et al. -The natural history of conservatively managed symptomatic intramedullary spinal cord cavernomas, Neurosurgery 2007, 60: 865-872.

7.Knipe H., Wein S. - Spinal cavernous malformation, Neurosurg Focus. 2010 Sep;29(3):E12

8.Ogilvy C.S. et al. - Intramedullary cavernous angiomas of the spinal cord: clinical presentation, pathological features and surgical management, Neurosurgery 1992, 31: 219-230

9.Park G.Y., Seo H.J. et al. - Intramedullary Cavernous Hemangioma as a Cause of Paraplegia: Two cases report, abstract, Journal of the Korean Academy of Rehabilitation Medicine 2002; 26(6): 815-818

10.Robinson J.R.et al. - Natural history of cavernous angioma, J. Neurosurg. 1991, 75, 709-714.

11.Grasso G. et al. - Thoracic spinal cord cavernous angioma: a case report and review of the literature, Journal of Medical Case Reports 2014, 8:271 doi:10.1186/1752-1947-8-271

12.Nagib M.G. et al. - Intramedullary cavernous angiomas of the spinal cord in the pediatric age group: a pediatric series. Pediatr. Neurosurg 2002, 36; 57-63;

13.Noudel R. et al. - Intramedullary spinal cord cavernous angioma in children: case report and literature review.Childs Nerv. Syst 2008, 24, 259-263.

14.Hatiboglu M.A. et al. - Epidural Spinal Cavernous Hemangioma Case report, Neurol Med Chir (Tokyo) 2006, 46, 455-458.

15.Zevgaridis D. et al. - Cavernous hemangiomas of the spinal cord. A review of 117 cases, Acta Neurochir. (Wien), 1999, 141: 237-245 
DOI: 10.2478/romneu-2014-0056

16.Iacob G. Olarescu A - Spinal Intramedullary Cavernomas.Personal experience reffering to six cases, Romanian Neurosurgical Congress Bucharest 2014 17.Harrison M.J. et al. - Symptomatic cavernous malformations affecting the spine and spinal cord Neurosurgery 1995, 37: 195-205.

18. Vishteh AG, et al. - Surgical resection of intramedullary spinal cord cavernous malformations: delayedcomplications, long-term outcomes and association with cryptic venous malformations. Neurosurgery 1997, 41:1094-1100

19.Jellinger K: Vascular malformations of the central nervous system: a morphological overview, Neurosurg Rev 1986, 9:177-216.

20.Rigamonti D, et al. - Cerebral venous malformations, J Neurosurg 1990, 73:560-564

21.Young K.A, Min Jeong M.H et al. - A Case of Spinal Cord Cavernoma Mimicking Transverse Myelitis, Case report, Abstract, Journal of the Korean Child Neurology Society 2010; 18(1) : 153-157

22.Miyoshi $\mathrm{Y}$, et al. - Infantile cervical intramedullary cavernous angioma manifesting as hematomyelia. Case report, Neurol Med Chir (Tokyo) 2010, 50:677-682

23.Bian LG, et al. - Intramedullary cavernous malformations: clinical features and surgical technique via hemilaminectomy, Clin Neurol Neurosurg 2009, 111:511-517

24.Deutsch H. et al. - Spinal intramedullary cavernoma: clinical presentation ans surgical outcome, J. Neurosurg. 2000, 93, 65-70

25.Sandalcioglu I.E. et al. Intramedullary spinal cord cavernous malformations; clinical features and risk of hemorrhage Neurosurg. Rev. 2003, 26: 253-256
26.Popescu M. et al. - Cauda Equina Intradural Extramedullary Cavernous Haemangioma: Case Report and Review of the Literature, Neurol MedChir (Tokyo) 2013, 53, 890-895

27.Weinzierl MR, et al. - MRI and intraoperative findings in cavernous haemangiomas of the spinal cord, Neuroradiology 2004, 46:65-71

28.Huffmann B.C. et al. - Treatment strategies and results in spinal vascular malformations, Neurol Med Chir Suppl (Tokyo) 1998, 38, 231-237

29.Badhiwala J.H. et al. - Surgical outcomes and natural history of intramedullary spinal cord cavernous malformations: a single-center series and meta-analysis of individual patient data, Journal of Neurosurgery: Spine, July 25, 2014; DOI: 10.3171/2014.6.Spine13949.

30.Gross B.A., et al. - Intramedullary spinal cord cavernous malformations, .Neurosurg Focus 2010, 29:E14.

31.Jallo G.I - Clinical presentation and optimal management for intrameullary cavernous malformations Neurosurg. Focus 2006, 21(1): e10

32.Cho Y.J. et al. - Surgical Treatment of Intramedullary Spinal Cord Cavernous Malformation, Abstract, Journal of Korean Neurosurgical Society 2003; 33(5): 466-471 33.Matsuyama Y. et al. - Surgical results of intramedullary spinal cord tumor with spinal cord monitoring to guide extent of resection.J Neurosurg Spine 2009, 10:404-413

34.Labauge P. et al. - Outcome in 53 patients with spinal cord cavernomas, Surg Neurol 2008, 70:176-181

35.Knipe H., Wein S. - Spinal cavernous malformation, Neurosurg Focus. 2010 Sep;29(3):E12. 